\title{
Introduction Se mobiliser en tant que musulmans. Condition minoritaire et engagement politique
}

\author{
Julien TALPIN, Franck FREgosi, Julien O’MIEL
}

\begin{abstract}
«M. Ries et son équipe ont fait une remarquable campagne de communication sur la démocratie de proximité, mais au-delà des mots qu'y a-t-il vraiment ? (...) Vous êtes des sous-hommes, vous êtes des sous-citoyens! Vous n'êtes pas des citoyens français! M. Ries vous considère comme des cafards et comme des rats! Restez dans votre cave ! Restez dans la rue ! Vous êtes des SDF! Vous n'êtes pas des citoyens français! Voilà ce que $M$. Ries vous envoie comme message ${ }^{1} \gg$.
\end{abstract}

L'islam a été constitué, au moins depuis les années 1980, comme un problème public². Les controverses à propos de l'islam sont légion : qu'il s'agisse de qualifier le port du foulard de "revendication communautaire», de (sur)déterminer le poids en son sein de courants religieux littéralistes (néo-salafisme) ou d'éléments politiquement radicaux (mouvance jihadiste ou takfiriste), ou de chercher à déceler les points de divergence ou de tensions entre certaines pratiques et la règle de laïcité. Ces controverses ont contribué à diffuser l'idée selon laquelle les musulmans - pensés comme une catégorie homogène - seraient difficiles à intégrer dans les pays occidentaux, voire qu'ils incarneraient un danger pour les valeurs démocratiques et le lien social ${ }^{3}$. Les actes terroristes et la visibilité de certains groupes rigoristes symboliseraient la menace qui pèse sur les sociétés européennes. Ces débats ont contribué à l'essentialisation - certains parlent de «racialisation ${ }^{4}-$ de la

${ }^{1}$ Propos d'un représentant d'une association de musulmans strasbourgeoise, suite au refus de la municipalité d'octroyer un terrain public en vue de la construction d'une mosquée, in Donnet (C.) «Une mosquée pour exister. Le militantisme de jeunes musulmans strasbourgeois », Archives de sciences sociales des religions, 2013, 162 (2), p. 219-234.

${ }^{2}$ Hajjat (A.), Mohamed (M.), Islamophobie. Comment les élites françaises fabriquent le "problème musulman », La Découverte, Paris, 2013. On pourrait en effet remonter bien plus loin, avec Edward Saïd notamment, voir L'orientalisme, Paris, Seuil, 1978.

${ }^{3}$ Deltombe (T.), L'islam imaginaire. La construction médiatique de l'islamophobie en France, 1975-2005, Paris, La Découverte, 2005.

${ }^{4}$ Meer (N.), Modood, (T.), "The multicultural state we're in: Muslims, 'multiculture', and the Civic rebalancing of British multiculturalism", Political Studies, 57(1), 2009, p.473-479; Voir le numéro spécial "Racialization and Religion: Race, culture and difference in the study of Antisemitism and Islamophobia", Ethnic and Racial Studies, vol. 36, n 3, 2013 ; Guénif Souilamas (N.), « La Française voilée, la beurette, le 
catégorie de «musulmans». Autrefois qualifiées dans des termes légaux ou ethniques comme des "immigrés», des «maghrébins» ou des "arabes » pour ne prendre que les catégories les plus fréquentes - certaines fractions de la population sont de façon croissante relabellisées sous l'étiquette de «musulmans $»^{5}$. La perception dominante qui prévaut dans les sociétés occidentales renvoie d'une part à celle d'un groupe social supposé en accroissement démographique, dénoncé parfois comme une "minorité tyrannique ${ }^{6}$, et appréhendé d'autre part à l'aune du seul prisme de la religion. Il semble à ce titre salutaire de suivre Roger Brubaker lorsqu'il invite à percevoir les "musulmans » non comme un groupe homogène et solidaire, mais comme une catégorie hétérogène, sujette à des luttes symboliques et politiques ${ }^{7}$.

De ces constructions essentialisantes de l'islam découlent la crainte, la haine ou l'hostilité à l'égard des musulmans, qualifiées d'islamophobie 8 . Il s'agit à la fois d'un ensemble de discours et d'attitudes, et de leurs effets pratiques, entrainant exclusion et discriminations. Chris Allen distingue ainsi l'islamophobie comme processus et comme produit ${ }^{9}$. Le processus s'appuie sur des stéréotypes, attribuant certains traits figés à des groupes spécifiques. Des recherches indiquent ainsi la croissance de la perception négative de l'islam ces dernières années ${ }^{10}$. Ces éléments symboliques ont également des conséquences pratiques - l'islamophobie comme produit - l'adhésion perçue ou supposée à l'islam se traduisant notamment par une discrimination très nette sur le marché du travail ${ }^{11}$.

Comment les fidèles réagissent-ils à ce cadrage du " problème musulman » ? Il s'agit non seulement de comprendre " ce que cela fait d'être un problème » ${ }^{12}$, mais plus largement, audelà des questions proprement symboliques, comment la condition minoritaire façonne le rapport au politique et à l'action collective des musulmans européens. Alors que la religion a $\mathrm{au}$ cours de l'histoire constitué un puissant ferment de politisation et d'engagement, notamment pour les fractions dominées de la société, qu'en est-il pour les musulmans européens, placés dans une situation minoritaire ? Il s'agit ici de banaliser et refroidir ces

garçon arabe et le musulman laïc. Les figures assignées du racisme vertueux », in Guenif-Souilamas (N.), dir., La République mise à nu par son immigration, Paris, La Fabrique, p. 109-132, 2005.

${ }^{5}$ Amiraux (V.) «Visibilité, transparence et commérage: de quelques conditions de possibilité de l'islamophobie... et de la citoyenneté », Sociologie, 2014, 5(1), p. 81-95.

${ }^{6}$ Zarka (Y C), «L'islam en France : vers la construction d'une minorité tyrannique ?», in Cités, Hors-série, L'islam en France, Puf, 2004, p. 1-4.

${ }^{7}$ Brubaker (R.) "Categories of analysis and categories of practice: A note on the study of Muslims in European countries of immigration", Ethnic and Racial Studies, 2013, 36(1), p. 1-8.

${ }^{8}$ Geisser (V.), La nouvelle islamophobie, Paris, La Découverte, 2003 ; Goldman (H.), Le rejet français de l'islam. Une souffrance républicaine, Paris, Puf, 2012.

${ }^{9}$ Allen (C.), Islamophobia, Farnham, Burlington, Ashgate, 2010. À ce sujet cf. aussi Asal, (H.), 2014, Hajjat (A.), Mohamed (M.), Islamophobie, op. cit.

${ }_{10}^{10}$ Pour la France cf. Brouard (S.), Tiberj (V.), 2006 ; Mayer (N.), Michelat (G.), Tiberj (V.), « Montée de l'intolérance et perception anti-islam » in Commission nationale consultative des droits de l'homme, La lutte contre le racisme et la xénophobie. Paris, La Documentation française, 2012, p. 28-47. ; pour d'autres pays, Morgan (G.), Poynting, (S.), Global Islamophobia Muslims and Moral Panic in the West, London, Ashgate, 2012, Nussbaum (M. C.), Les religions face à l'intolérance. Vaincre la politique de la peur, Paris, Climats, Flammarion, 2013.

${ }^{11}$ Adida (C.), Laitin (D.), Valfort (M.-A.), Les Français musulmans sont-ils discriminés dans leur propre pays? Une étude expérimentale sur le marché du travail, New York/Paris, French American Foundation/Sciences Po, 2010.

${ }^{12} \mathrm{Arab}$ (C.) et al. "Qu'est-ce que ça fait d'être un problème ? ", Mediapart, 21 janvier 2015. Les auteurs reprennent ici le titre d'un célèbre essai de W.E.B. Du Bois au sujet de la question noire aux États-Unis. https://blogs.mediapart.fr/edition/les-invites-de-mediapart/article/210115/qu-est-ce-que-ca-fait-detre-un-probleme 
questions qui font souvent l'objet de vives polémiques dans le débat public. Cela implique de s'intéresser à la façon dont des groupes d'individus de confession musulmane sont amenés, dans le cadre de mobilisations collectives et d'actions politiques souvent locales, à mettre en avant une appartenance héritée ou une affiliation choisie à l'islam. Celui-ci peut alors être envisagé comme une ressource symbolique dont les acteurs tirent des raisons d'agir, des motivations, un vocabulaire et des récits leur permettant de se faire entendre pour contester, revendiquer et penser leur situation sociale. Nous chercherons ici à retracer la façon dont les sciences sociales se sont saisies des rapports entre religion et engagement militant, avant de nous pencher sur la littérature s'attaquant spécifiquement aux rapports des musulmans européens à l'action collective. Il convient auparavant de préciser les usages que nous faisons - ici et tout au long de cet ouvrage - de la catégorie de « musulmans ».

\section{L'islam n'est qu'un mot : diversité des pratiques religieuses et pluralité des appartenances}

Sans prétendre dresser un état des lieux exhaustif des différents modes d'appréhension des interactions entre religion et politique dans les diverses branches de la science politique (sociologie historique, sociologie des mobilisations, politique comparée, sociologie du vote...), il importe toutefois de dissiper d'éventuels malentendus quant à ces formes d'engagement et de mobilisations collectives. Le fait que dans une société profondément laïcisée comme la société française, des personnes engagées dans diverses formes contemporaines de mobilisation fassent ponctuellement et de façon disparate référence à l'islam pourrait, au mieux, surprendre par son côté "anachronique » en régime de sécularisation avancée, ou pire, conduire à postuler une approche mécanique des rapports entre appartenance religieuse à l'islam et engagement politique dans la cité. Ces mobilisations seraient révélatrices d'une certaine idéalisation du modèle républicain classique d'intégration censé reléguer dans l'espace privé les affiliations religieuses. ${ }^{13}$ Afin d'éviter de tels raccourcis, il convient de préciser que s'intéresser aux effets sociaux des croyances sur les comportements politiques des acteurs, comme questionner les formes plurielles de participation politique de populations croyantes, voire étudier le recours à des représentations religieuses comme mode de dépassement d'une fragilisation sociale, n'enlève rien au fait que les populations musulmanes expérimentent également les effets de cet environnement sécularisé. Cela se vérifie notamment par le fait que la pratique régulière de la religion musulmane n'est pas aussi massive qu'on l'imagine, et que les musulmans - à l'instar de ce que l'on peut observer au sein d'autres groupes confessionnels - sont aussi concernés par un processus d'individualisation du croire. Ce processus les amène dès lors à reconsidérer de façon très pragmatique leur rapport à la lettre de la religion, à composer avec ses impératifs, à repenser les catégories de la pensée et de la loi islamique à l'aune de leur situation de minoritaire ${ }^{14}$ mais également, pour une fraction d'entre eux, à revendiquer une certaine forme de sortie de la religion elle-même ${ }^{15}$. Les musulmans français sont donc à la fois sujets et objets de leur propre sécularisation ${ }^{16}$. Si l'islam renvoie d'abord à une

\footnotetext{
${ }^{13}$ Cf. Baubérot (J.), Milot (M.), Laïcités sans frontières, Paris, Seuil, La couleur des idées, 2011.

${ }^{14}$ Bowen (J. R.), Can Islam be French ? Princeton, Princeton University Press, 2010 ; Frégosi (F). «Usages sociaux de la référence à la charia chez les musulmans d'Europe » in Dupret (B.), (ed.) La charia aujourd'hui. Usages de la référence au droit islamique, Paris, La Découverte, Coll. Recherches, 2012, p. 6577.

${ }^{15 B i d a r}$ (A.), L'islam sans la soumission. Pour un existentialisme musulman, Paris, Albin Michel, coll. «L'islam des lumières », 2008.

${ }^{16}$ Frégosi (F.), « Musulmans en Europe occidentale. Spectateurs et acteurs de la sécularisation de l'islam », in Vaner (S.) Heradstveit (D.) Kazancigil (A.), dir., Sécularisation et démocratisation dans les sociétés musulmanes, Bruxelles, Peter Lang, Coll. Dieux, Hommes et Religions, $\mathrm{n}^{\circ}$ 15, 2008, p 261-284.
} 
expérience religieuse, celle-ci est pour le moins hétérogène tant au regard de la diversité de son expression, que par les nombreux courants et sensibilités qui s'en réclament ${ }^{17}$.

Que faut-il entendre par mobilisations de musulmans? S'agit-il de cibler des mobilisations explicitement islamiques dès lors perçues comme une effusion du religieux hors des murs des mosquées et des salles de prière en prenant le risque de reprendre à son compte une supposée spécificité ontologique de l'islam, religion réputée plus politisée que d'autres? S'agit-il plutôt de distinguer des mobilisations strictement musulmanes, autrement dit confessionnelles ou religieuses, dont la finalité serait d'assurer le rayonnement, la promotion de l'islam comme religion et son substrat pratique (mosquées, prières, pratiques dévotionnelles...) ? S'agit-il plus simplement de situations dans lesquelles des acteurs sociaux inégalement dotés en capital social et/ou militant, confrontés à une situation d'injustice sociale avérée ou ressentie comme telle et inscrit dans une quête de reconnaissance ou de concrétisation d'un projet précis, puisent dans l'islam les ressources nécessaires pour légitimer leur action dans l'espace public ? Les enquêtes laissent entendre qu'en agissant de la sorte au nom de l'islam, les acteurs cherchent autant à s'adresser à leurs coreligionnaires auprès desquels ils espèrent écoute et soutien (social, matériel, militant, symbolique...) voire briguer leurs suffrages, qu'au reste de la société française, pour rappeler leur légitime présence et leur commune citoyenneté. Il est donc légitime de parler avant tout de mobilisations de musulmans (au pluriel).

Les pages qui suivent invitent à appréhender ces formes d'engagement politiques au nom de l'islam comme autant de mises en scène des rapports complexes que les musulmans entretiennent avec l'islam comme religion et comme culture. Il serait d'ailleurs plus judicieux de raisonner en termes d'islamité, dans le sens où à l'instar de la judéité ou de la " chrétienté »18, il s'agit de pointer l'auto-perception que se font les acteurs sociaux de leur identification confessionnelle et de leurs rapports symboliques et pratiques avec la religion, la façon dont ils recomposent leurs relations à celle-ci selon des modes plus ou moins religiocentrés, culturels voire séculiers. Si une certaine compréhension théologique homogène, plutôt orthodoxe et conservatrice de l'islam, semble néanmoins se dégager des différents groupes de musulmans étudiés ici, le poids du contexte et plus en amont les socialisations et les diverses positions sociales occupées par les individus contribuent tout autant à influer sur leurs comportements politiques et rendent compte d'usages sociaux différenciés d'une islamité résolument plurielle.

Il n'y a dès lors pas lieu de reprendre à notre compte la représentation des musulmans comme un sujet collectif constitué par un ensemble homogène de populations identifiées par une commune religion supposée uniformément vécue et pratiquée. Cette approche nous condamnerait à ne pas appréhender d'autres modes de compréhension des engagements de ces populations, qui, pour être certes de confession musulmane, n'ont de celle-ci ni la même compréhension, ni la même intensité dans la pratique, ni ne réduisent l'islam à un simple identifiant confessionnel ${ }^{19}$. Lesdits musulmans ne sont pas que musulmans. Il va de soi que les individus disposent d'une pluralité d'identités, pouvant se définir selon des caractéristiques de classe, de genre, d'ethnicité, des référents politiques, sociaux ou territoriaux, mais aussi selon leur affiliation religieuse. Si la population musulmane en Europe est hétérogène, étant composée de nationaux et d'étrangers, de personnes issues d'une immigration originaire de sociétés dont l'islam est la religion majoritaire comme de

\footnotetext{
${ }^{17}$ Frégosi (F.), «Formes de mobilisation collective des musulmans en France et en Europe », in Revue Internationale de Politique Comparée, Vol 16, n² 1, 2009, p. 41-61.

${ }^{18} \mathrm{Chrétienté} \mathrm{est} \mathrm{à} \mathrm{entendre} \mathrm{ici} \mathrm{non} \mathrm{comme} \mathrm{le} \mathrm{fait} \mathrm{de} \mathrm{renvoyer} \mathrm{à} \mathrm{un} \mathrm{ensemble} \mathrm{historique,} \mathrm{humain,} \mathrm{territorial}$ et symbolique caractérisé par l'affiliation au christianisme, mais comme le fait de s'adosser à titre individuel à une identification au christianisme sans nécessairement que celle-ci débouche sur une pratique religieuse avérée.

${ }^{19}$ Frégosi (F,), L'islam dans la laïcité, Paris, Pluriel Hachette, 2011.
} 
personnes ayant choisi cette religion (convertis), la majorité fait partie des classes populaires ${ }^{20}$. Quelles sont les conséquences de cette position spécifique dans la hiérarchie sociale ? Comment, à quelles conditions et dans quels contextes des individus et des groupes se saisissent ou non de leur identité religieuse, ici musulmane, pour s'engager dans l'espace public?

\section{Penser la condition minoritaire}

Cette question se pose de façon spécifique dans le contexte européen où la religion musulmane est minoritaire, pratiquement et symboliquement. L'essentialisation de la catégorie de "musulmans » a contribué à façonner cette "condition minoritaire », qui « désigne une situation sociale qui n'est ni celle d'une classe, d'un État, d'une caste ou d'une communauté, mais d'une minorité, c'est-à-dire d'un groupe de personnes ayant en partage, nolens volens, l'expérience sociale d'être généralement considérées ${ }^{21}$ » comme musulman. La façon dont le "problème musulman » a été historiquement construit en Occident a contribué à façonner des expériences sociales spécifiques, minoritaires ${ }^{22}$. Ce qui unit les acteurs dans ce cadre ne serait donc pas tant la « communauté », puisque les façons de vivre et pratiquer l'islam varient, que «l'expérience minoritaire», définit comme "l'assujettissement à une relation de pouvoir " ${ }^{23}$. À ce titre, nous cherchons à analyser ici ce que la condition minoritaire fait aux formes d'engagement et aux mobilisations collectives. En particulier, la condition minoritaire, qui induit des formes plus ou moins institutionnalisées de stigmatisation, de discrimination et de domination, peut se traduire par des dynamiques spécifiques de retournement du stigmate, de mises en avant de son identité minorisée ou à l'inverse d'invisibilisation. Ces processus se déploient différemment en contexte majoritaire. Ici, ces mobilisations peuvent viser à définir ou transformer cette condition minoritaire. Il convient en particulier de saisir quels sont les processus, sociaux, politiques et discursifs, par lesquels une expérience sociale de minoration en vient ou non à être conçue comme telle et partagée par un groupe porteur d'intérêts communs. Quels sont les mécanismes et les acteurs de ces processus de montée en généralité et de politisation? Comment l'expérience minoritaire devient-elle (ou non) un critère d'unification d'un groupe qui peut être traversé par de nombreux clivages ? Comment est-elle mobilisée, vis-à-vis de l'extérieur, comme un critère de légitimation des demandes du groupe ?

Ces questions nous semblent d'autant plus importantes qu'en dépit d'un intérêt important des sciences sociales pour l'islam depuis une trentaine d'années, peu de travaux ont directement portés sur les conditions de mobilisation de musulmans en situation minoritaire ${ }^{24}$. Si des travaux existent sur les mobilisations de musulmans en Amérique du

\footnotetext{
${ }^{20}$ Dargent (C.), «Les musulmans déclarés en France: affirmation religieuse, subordination sociale et progressisme politique » Les cahiers du Cevipof, 2003, 34, p. 2-42.

${ }^{21}$ Ndiaye (P.), La condition noire. Essai sur une minorité française, Paris, Seuil, 2009, p. 29.

${ }^{22}$ Jounin (N.), Palomares (É.), Rabaud (A.), «Éthnicisations ordinaires, voix minoritaires 》, Sociétés contemporaines, 2008, 70 (2), p. 7-23.

${ }^{23}$ Fassin (D.), Fassin (E.), «Conclusion. Éloge de la complexité », in De la question sociale à la question raciale. Représenter la société française, Paris, La Découverte, 2006. Nous suivons en ce sens Hajjat (A.) et Mohammed, (M.) quand ils avancent: "L'appartenance religieuse, rappelons-le minoritaire, constitue bien le vecteur potentiel d'une condition collective dans la mesure où elle transcende les marqueurs ascriptifs (sexe, âge, origine) et l'appartenance de classe. La thématique de l'islamophobie (...) symbolise cette exposition collective au rejet social et participe du coup de la construction de la condition musulmane », in Islamophobie, op. cit., p. 64.

${ }^{24}$ Comme l'indique Amiraux (V.), «État de la littérature. L'islam et les musulmans en Europe : un objet périphérique converti en incontournable des sciences sociales ", Critique internationale, 2012, (3), p. 141157 ; Voir également Cesari (J.), Mc.Loughlin (J.), dir., European Muslims and the Secular State, Aldershot,
} 
Nord, au Royaume-Uni ${ }^{25}$, dans les pays scandinaves ou aux Pays-Bas, ils sont peu nombreux dans le cas français. Une raison de cette absence est peut-être la difficulté, la faiblesse, la discrétion ou l'invisibilité de l'action collective de groupes musulmans en France en tant que tels. Quelles sont dès lors les conditions sociales de l'engagement de groupes souvent dénués de ressources sociales et politiques? Dans quelle mesure la structure des opportunités politiques et discursives, variable selon les pays, contraint ces formes d'action collective $^{26}$ ? C'est ainsi autant les mobilisations que leurs conditions d'émergence ou leur absence dans différents espaces locaux et nationaux qui nous intéressent ici. Quand la mobilisation prend, quels types de revendications sont alors mises en avant? L'action collective de musulmans vise-t-elle à la reconnaissance symbolique de ces groupes ? Se mobiliser sur des bases religieuses signifie-t-il porter des revendications exclusivement cultuelles ou confessionnelles (construction de mosquées, nourriture halal, port du hijab, etc.) ? Alors que la littérature portant sur les liens entre religion et engagement politique insiste sur la transitivité qui irait de l'une à l'autre, qu'en est-il pour les religions minoritaires, et ici de l'islam ? Nous chercherons à interroger les réponses apportées par les sciences sociales à ces questions afin de dégager certains chantiers de recherche qu'il nous semble urgent d'explorer. En amont de ces questions, il convient de rappeler combien l'incidence sociale du religieux dans les dynamiques de mobilisation constitue une question qui traverse les sciences sociales.

\section{Le religieux dans les dynamiques de contestation : un classique des sciences sociales}

S'interroger sur les interactions entre pratiques religieuses et comportements sociaux, et entre autres les comportements politiques, est un grand classique de la sociologie. Tant Durkheim que Weber ont pointé le rôle social de la religion et ses multiples incidences sociales en tant que prédisposition à l'action. Dans les formes élémentaires de la vie religieuse, Durkheim démontre d'abord que la religion n'est que l'hypostase de la société, en d'autres termes que derrière la religion et ses cérémonies il n'y a rien d'autre que la société, une représentation idéalisée du collectif englobant ${ }^{27}$. Mais le fondateur de l'école française de sociologie reconnaît en même temps que par le biais d'activités religieuses spécifiques (culte, dévotions quotidiennes, transe, etc.), l'homme entré en dialogue avec le divin semble y puiser des forces pour agir efficacement : « Le fidèle qui communie avec son dieu n'est pas seulement un homme qui voit des vérités nouvelles que l'incroyant ignore; c'est un homme qui peut davantage. Il sent en lui plus de force soit pour supporter les difficultés de

Ashgate, 2005 ; Frégosi (F.), «Formes de mobilisation collective des musulmans en France et en Europe », op. cit.; De Galembert (C.), "Cause du voile et lutte pour la parole musulmane légitime », Sociétés contemporaines, 2009, (2), p. 19-47 ; Sinno (A. H.), " The Politics of Western Muslims », Review of Middle East Studies, 2012, 46(2), p. 216-231; Monnot (C.), (dir.), La Suisse des mosquées. Derrière le voile de l'unité musulmane, Geneve, Labor et Fides, 2013 ; Nielsen (J.), dir., Muslim political participation in Europe, Edimbourg, Edimbourg University Press, 2013.

${ }^{25}$ Bolognani (M.), Statham (P.), "The changing public face of Muslim associations in Britain: Coming together for common 'social' goals?", Ethnicities, 2013, 13 (2), p. 229-249 ; Dobbernack (J.), Meer (N.), Modood (T.), "Misrecognition and Political Agency. The Case of Muslim Organisations in a General Election", The British Journal of Politics \& International Relations, 2015, 17 (2), p. 189-206.

${ }^{26}$ Cinalli (M.), Giugni (M.), "Political opportunities, citizenship models and political claim-making over Islam”, op. cit.; Carol (S.), Koopmans (R.), "Dynamics of contestation over Islamic religious rights in Western Europe”, Ethnicities, 2013, 13(2), p. 165-190.

${ }^{27}$ Durkheim (É.), Les formes élémentaires de la vie religieuse. Le système totémique en Australie, Presses universitaires de France, coll. «Quadrige Grands textes », 2008. 
l'existence soit pour les vaincre ${ }^{28}$. » Quant à Weber, il n'a eu de cesse dans ses écrits de démontrer que la religion dont il récusait toute définition essentialiste, n'est qu'une espèce particulière d'agir en société dont il faut étudier les conditions et les effets en partant des significations que les individus donnent à leurs pratiques religieuses (expériences subjectives, représentations, fins poursuivies). Parmi ses apports décisifs figure notamment celui de nous inciter à l'analyse des affinités électives entre une certaine éthique religieuse de vie (celle du protestantisme ascétique et puritain) et l'esprit d'entreprise du capitalisme, autrement dit l'existence d'une transitivité entre des formes de croyances et des comportements économiques précis, mais sans jamais prétendre que le capitalisme est mécaniquement fils du protestantisme. Il a enfin participé à une analyse précise des formes d'interactions entre le pouvoir religieux (la hiérocratie) et le pouvoir séculier temporel et démontré combien la religion constituait une ressource symbolique de légitimation des pouvoirs en place et un outil de domestication des dominés ${ }^{29}$.

L'Église chrétienne en Occident, quand elle ne revendiquait pas pour elle-même la suprématie absolue du pouvoir, a bien souvent été utilisée comme une courroie de transmission du pouvoir civil remplissant en cela une fonction de légitimation de l'ordre dominant. Il convient néanmoins d'historiciser l'analyse afin de ne pas réifier l'appréhension du religieux, en en faisant partout et tout le temps le suppôt du pouvoir. L'histoire sociale est traversée par des mobilisations qui ont pour tout ou partie un support religieux $^{30}$. Bien que l'histoire des révoltes et des mouvements sociaux depuis le XIX ${ }^{\mathrm{e}}$ siècle en France ${ }^{31}$ et en Europe semble confirmer le caractère résolument séculier de ces mobilisations et l'absence de toute référence confessionnelle (mais pas le défaut de ritualisation ${ }^{32}$ ), il n'en a pas toujours été ainsi. "La détresse religieuse écrit Marx est, pour une part, l'expression de la détresse réelle et, pour une autre, la protestation contre la détresse réelle ${ }^{33}$. » Marx et ses disciples ont joué un rôle central dans la déconstruction et la dénonciation du fait religieux comme bonheur illusoire et imposture sociale ${ }^{34}$. Relevons cependant qu'une lecture plus fine de leurs écrits révèle en creux que la religion pouvait, y compris dans une optique marxiste, être autre chose qu'une pure illusion sociale et nourrir des formes de protestation dont le mouvement ouvrier devait pouvoir tirer des leçons ${ }^{35}$. C'est ce que tentent par exemple de montrer plus tardivement les approches dissidentes d'Antonio Gramsci, d'Ernst Bloch ${ }^{36}$ et de Karl Manheim ${ }^{37}$, sans parler aujourd'hui de l'étonnante réhabilitation de l'héritage chrétien opérée par Slavoj Žižek pour contrer la déferlante de spiritualités post-modernes ${ }^{38}$.

\footnotetext{
${ }^{28}$ Ibid., p 693-694.

${ }^{29}$ Weber (M.), La domination, Paris, La Découverte/Politique et Sociétés, 2013.

${ }^{30}$ Lavignotte (S.), Les religions sont-elles réactionnaires ? Paris, Textuel, 2014.

${ }_{31}^{31}$ Pigenet (M.), Tartakowsky (D.), dir., Histoire des mouvements sociaux en France, Paris, La Découverte, 2014.

32Fureix (E.), La France des larmes. Deuils politiques à l'âge romantique (1814-1840), Paris, Epoques, Champ Vallon, 2009.

${ }^{33}$ Marx (K.), « Critique de la philosophie du droit de Hegel », in Marx (K.), Engels (F.), Sur la religion, Paris, Éditions sociales, 1972, p 42.

${ }^{34}$ Quiniou (Y.), Critique de la religion. Une imposture morale, intellectuelle et politique, Paris, La ville brûle, 2014.

35Dianteill (E.), Lôwy (M.), Sociologies et religion. Approches dissidentes, Paris, PUF, Sociologie d'aujourd'hui, 2005.

36Bloch (E.), L'esprit de l'utopie, Paris, Gallimard, 1977 ; L'athéisme dans le christianisme, Paris, Gallimard, 1978.

37Mannheim (K.), Idéologie et utopie, Paris, Editions de la Maison des sciences de l'homme, 2006.

38Žižek (S.), Fragile absolu. Pourquoi l'héritage chrétien vaut-il d'être défendu?, Paris, Champs Essais, Flammarion, 2008.
} 
Il suffit de relire La Guerre des Paysans de Friedrich Engels pour se convaincre que la lutte sociale au XVIe siècle, dans une Europe ébranlée par la Réforme protestante et ses prolongements millénaristes, empruntait souvent le canal des querelles théologiques. " Il est donc clair, écrit Engels, que toutes les attaques dirigées en général contre le féodalisme devaient être avant tout des attaques contre l'Église, toutes les doctrines révolutionnaires, sociales et politiques, devaient être en même temps et principalement, des hérésies théologiques. Pour pouvoir toucher aux conditions sociales existantes, il fallait leur enlever leur caractère sacré. ${ }^{39}$ Aussi les guerres de religion entre les catholiques et les protestants comme celles entre les princes luthériens et les paysans plébéiens se revendiquant de Thomas Müntzer étaient-elles des luttes de classes. Leur vernis religieux s'expliquait par les conditions sociales de l'époque, par l'absence d'espace autonome par rapport à la prégnance du sentiment religieux et la puissance sociale de l'Église romaine. Face à une Église catholique alliée au pouvoir impérial et à la haute noblesse des villes allait se dresser « l'Église à bon marché » celle de la Réforme qu'appelaient de ses vœux la bourgeoisie des villes, la petite noblesse et une partie des princes séculiers. Mais face à celle-ci, devait également s'opposer celle des dominés (celle de Müntzer entre autres), regroupant des paysans désœuvrés qui non seulement réclamaient le rétablissement des conditions d'égalité du christianisme primitif mais qui passèrent à l'acte en retournant leurs armes contre les seigneurs protestants et les bourgeois des villes au nom d'un idéal aux fortes résonances communistes. Eric Hobsbawm scrutant en plein XIX siècle les formes élémentaires - au sens d'archaïques - d'agitation sociale (banditisme, mafias, émeutes paysannes et urbaines.... $)^{40}$ pointe également parmi une multitude de mouvements sociaux insurrectionnels l'effervescence de courants directement liés à des mouvements religieux à l'instar des Lazzarettistes toscans et des sectes ouvrières anglaises. Même si ces mouvements s'essoufflent progressivement à la fin du XIXe siècle, leur existence montre à l'évidence «un parallélisme marqué entre les mouvements religieux, sociaux et politiques $»^{41}$.

Plus près de nous, les travaux pionniers de Guy Michelat et Michel Simon ${ }^{42}$ sur l'incidence de la variable religieuse (appartenance et degré d'intégration religieuse) sur les comportements politiques, notamment électoraux, les recherches de Jean Marie Donegani ${ }^{43}$ sur les effets politiques pluriels des croyances religieuses et plus directement encore ceux de Jacques Lagroye ${ }^{44}$ sur l'Eglise catholique ont montré comment les croyances, l'adhésion à des régimes de vérité, les modes d'appartenance à une institution religieuse sont susceptibles de nous renseigner sur les différents points d'ancrage du religieux sur l'individu. Aussi, la question des incidences sociales et politiques du religieux (cléricalisme électoral, religion et engagement politique, religion et politisation...) ${ }^{45}$ sur les dynamiques

${ }^{39}$ Engels (F.), «La guerre des paysans », in Marx (K.), Engels (F.), Sur la religion, Paris, Éditions sociales, 1972, p. 100.

${ }^{40}$ Hobsbawm (E.), Les primitifs de la révolte dans l'Europe moderne, Paris, Pluriel Hachette, 2012.

${ }^{41}$ Ibid., p. 195.

${ }^{42}$ Michelat (G.), Simon (M.), Classe, religion et comportement politique, Presses de la FNSP et éditions sociales, 1977.

${ }^{43}$ Donegani (J.-M.), La liberté de choisir, Paris, Presses de la Fondation nationale des sciences politiques, 1993.

${ }^{44}$ Lagroye (J.), Appartenir à une institution. Catholiques en France aujourd'hui, Paris, Economica, Collections Études politiques, 2009 ; La vérité dans I'Église catholique. Contestations et restauration d'un régime d'autorité, Paris, Belin, 2006.

${ }^{45}$ Deloye (Y.), Les voix de Dieu. Pour une autre histoire du suffrage électoral : le clergé catholique français et le vote, XIXe-XXe siècle, Paris, Fayard, 2006 ; Della Suda (M.), « La politique malgré elles. Mobilisations féminines catholiques en France et en Italie (1900-1914) », in RFSP, Vol 60, n 1, 2010, p 37-60 ; Raison Du Cleziou (Y.), «Le couvent comme dispositif d'imposition de la vérité religieuse. Orthopraxie et 
de mobilisation et notamment sur les parcours de politisation des acteurs n'est pas une question secondaire ou une simple variable d'ajustement. Elle contribue notamment activement à penser la politique informelle ${ }^{46}$. Les travaux de sociohistoire sont nombreux à étudier le rôle de l'Église dans l'apprentissage du jeu démocratique ${ }^{47}$, les dynamiques d'éducation populaire initiés par des chrétiens de gauche ${ }^{48}$ ou la façon dont des dispositions acquises lors de la socialisation religieuse sont parfois reconverties dans le champ politique ${ }^{49}$. On manque néanmoins à ce jour de travaux aussi fins concernant la transitivité (ou non) de la socialisation musulmane à l'engagement politique en contexte minoritaire ${ }^{50}$. Il s'agit non seulement d'opérer à un niveau microsociologique - à l'échelle des trajectoires individuelles - qu'à un niveau méso-sociologique, en étudiant le rôle de certaines institutions et organisations religieuses (mosquées, associations, etc.), à l'image des travaux précédemment cités sur les catholiques en France, ou les baptistes dans le mouvement des droits civiques aux États-Unis ${ }^{51}$.

\section{Saisir les croyances au concret}

Le parti pris de cet ouvrage, écrit peu avant que Paris et ses alentours, sans oublier Nice et Saint Etienne du Rouvray, ne soient pris pour cible par des jihadistes, est donc de saisir les logiques et ressorts de mobilisation ordinaires de musulmans en se défaisant d'une approche essentialiste du religieux qui renverrait à une alternative manichéenne : ces mobilisations seraient religieuses ou ne le serait pas. Autrement dit, il s'agit ici de comprendre ces mobilisations en observant la façon dont le religieux est ou non invoqué dans les pratiques concrètes des acteurs. Cette approche du religieux par le bas permet alors d'appréhender la multiplicité des rapports au religieux qu'engagent ces musulmans dans le cadre de mobilisations collectives.

Il ne s'agit donc pas de postuler ex abrupto que le religieux serait immuablement une matrice du politique ou qu'un politique raisonnablement profane n'est pas pensable hors de l'influence du religieux notamment parmi les musulmans de France. Il faut tout à la fois ne pas ignorer son influence, hier plus directe, aujourd'hui sans doute plus diffuse, dans certains groupes sociaux, et tâcher de raccorder celle-ci à des situations précises (opportunités politiques, configuration sociale, contexte régional et international), à des logiques d'intérêts et d'action politique (contestation, résistance, alliance, accession au pouvoir) selon des modalités sociales particulières (démocratique, régimes autoritaires,

orthodoxie dans la Province dominicaine de France durant l'après-guerre », Sociétés contemporaines, 88 (4), 2012, p. 73-98; "Albert-Marie Besnard, un maître dépassé par ses disciples. Mystique de la génération et subversion des formes de l'autorité au couvent d'étude dominicain du Saulchoir (19641968)», Genèses, n88, 2012.

${ }^{46}$ Le Gall (L.), Offerlé (M.), Ploux (F.), La politique sans avoir l'air. Aspects de la politique informelle, XIX ${ }^{e_{-}}$ XXe siècle, Rennes, PUR, Coll. Histoire, 2012.

${ }^{47}$ Della Suda, (M.), « La politique malgré elles », Revue française de science politique, 60 (1), 2010, p. 37 60.

48Pierrard (P.), Launay (M.), Trempé (R.), La JOC. Regards d'historiens, Paris, L'Atelier, Éditions ouvrières, 1989 ; Duriez (B.), Fouilloux (E.), Pelletier (D.), Viet-Depaule (N.), Les catholiques dans la République (1905-2005), Paris, Éditions de l'Atelier, 2005.

${ }^{49}$ Sur les matrices religieuses de l'engagement de certains soixante-huitards, cf. Pagis, (J.), « La politisation d'engagements religieux », Revue française de science politique, 60 (1), 2010, p. 61-89

50 Il en va évidemment tout autrement pour les pays où l'islam est la religion majoritaire. Voir par exemple sur l'Égypte, Vannetzel, (M.), « Affection, désaffection et défection chez deux jeunes Frères musulmans en Égypte », Critique internationale, 65 (4), 2014, p. 127-147.

${ }^{51}$ Morris, (A.), The Origins of the Civil Rights Movement: Black Communities Organizing for Change, New York, The Free Press, 1984. 
violence armée) mais sans sacrifier toute réflexion sociologique sur l'autel d'une hypothétique revanche du religieux. Ces précautions, loin d'être rhétoriques, ne visent pas pour autant à occulter la variable religieuse ${ }^{52}$ et à réduire son incidence sur les comportements politiques des individus. Elles découlent davantage d'une démarche résolument sociologique qui vise à reconnaître dans le religieux comme l'écrit Jacques Lagroye «un registre de croyances $»^{53}$ parmi d'autres qui permet aux acteurs sociaux de donner un sens à leurs conduites, à leurs engagements dans la société, à leurs détresses comme à leurs attentes.

L'apport majeur de l'étude $\mathrm{du}$ fait religieux par les sciences sociales réside définitivement, comme l'écrit Peter Berger, dans cet effort de localiser socialement les phénomènes religieux ${ }^{54}$, de ramener les idées et les comportements religieux à des situations sociales vécues par ceux qui les professent et qui les pratiquent. Il s'agit alors de chercher à rapporter ces affirmations, ces professions de foi, ces énoncés normatifs à des situations de "frustrations, des ambitions ou des ressentiments ancrés 55 » dans des parcours de vie, dans des carrières militantes et dans des dynamiques sociales précises et nullement de nous livrer à une opération de vérification du contenu plus ou moins pertinent d'un énoncé religieux.

Ce n'est pas parce que dans les pages qui suivent, nous ne nous sommes pas focalisés sur l'exégèse d'un ensemble plus ou moins cohérent de textes à forte résonnance religieuse que nous sommes réfractaires à toute prise en compte de l'influence latente des discours et des représentations religieuses sur lesquelles s'adossent certains des acteurs étudiés, comme répertoires de légitimation de mobilisation ciblées. Ce désir de saisir de l'intérieur les ressorts idéologiques et en l'espèce proprement «religieux» des mobilisations de musulmans étudiées, à partir d'un énoncé religieux ou d'un catéchisme théologique ne semble néanmoins pas être l'approche sociologique la plus pertinente pour analyser les interactions entre croyances religieuses et engagement politique ${ }^{56}$. Osons faire un parallèle avec la question de la radicalisation violente qui a frappé de plein fouet l'hexagone durant l'année 2015 et l'été 2016. La connaissance la plus fine des corpus doctrinaux mêlant un vocabulaire emprunté à l'histoire longue de l'islam (le Califat par exemple), une rhétorique théologique millénariste, des analyses politiques et des considérations géopolitiques sur les conflits qui minent le Proche Orient que mobilisent les théoriciens du jihad en Europe et en France en particulier afin de tenter de justifier « religieusement » le recours à la violence la plus directe, ne saurait suffire à comprendre le processus dit de "radicalisation » dans lequel se sont engagés certains jeunes musulmans européens qui les a conduit à prendre pour cible des caricaturistes, des juifs et des forces de l'ordre en janvier 2015, puis à s'en prendre en novembre 2015 à des consommateurs attablés à des terrasses de restaurants et des spectateurs d'un concert de rock, à une foule de personnes à Nice assistant aux festivités du 14 juillet, et à un prêtre catholique célébrant un office ${ }^{57}$. L'existence de corpus idéologiques légitimant le recours à la violence la plus directe, n'explique pas tout et surement pas le passage à l'acte. N'en déplaise à certains, les sciences sociales sont pleinement dans leur rôle quand elles cherchent à comprendre (sans nul désir d'excuser ni de justifier) ce qui a pu conduire des individus jeunes ayant des profils sociaux différents (milieux populaires, classes moyennes), venants d'horizons culturels distincts (familles

\footnotetext{
${ }^{52}$ Dargent (C.) «Religion et vote : "Cachez cette variable que je ne saurais voir"...», in http://www.cevipof.com/bpf/analyses/Dargent_Religions.pdf

${ }^{53}$ Lagroye (J.), op. cit., 2009, p. 33.

54Berger (P.), Invitation à la sociologie, Paris, La découverte, Coll. Classiques Grands repères, 2006.

${ }^{55}$ Ibid, p. 153.

${ }^{56}$ Lichterman (P.), "Religion in Public Action From Actors to Settings", Sociological Theory, 2012, 30(1), p. 15-36.

${ }^{57}$ Roy (0.), Le djihad et la mort, Paris, Seuil, 2016.
} 
d'origine musulmane, familles non musulmanes), certains ayant connu la délinquance, à s'engager dans des voies de "radicalisation ». Cela revient à soulever la question du poids des déterminismes sociaux à l'œuvre dans de telles situations, comme celui des contextes qui ont pu aussi s'avérer déterminants dans telles ou telles trajectoires individuelles de "radicalisation", voire la part de l'aléatoire. À nos yeux, la religion n'est pas mécaniquement ce que peuvent en dire les clercs et leurs textes ou ce que croient les fidèles, c'est avant tout un réservoir de pratique et une mise en scène de la société. Il s'agit d'une théâtralisation de la vie humaine avec son lot d'acteurs, de figurants, de machinistes et de metteurs en scène, se référant à un ensemble de récits et de règles plus ou moins codifiées. Notre cœur de métier, consiste à visiter les arrières scènes, à aller dans les coulisses, à découvrir la société qui se dissimule sous le costume du religieux, à lever le rideau des apparences et des évidences sociales.

$\mathrm{Au}$ fond, en nous intéressant à des situations précises, à des contextes militants et des moments de mobilisation qui sollicitent le phénomène religieux, nous entendons montrer que celui-ci est autant un dire, un croire, qu'un faire. Ce faisant nous cherchons à saisir les effets du rapport au religieux par le bas, par la façon dont s'en saisissent les acteurs en situation. En repérant les éventuelles occurrences religieuses dans les discours, les attitudes, les actions de militants associatifs de quartiers populaires, de femmes voilées s'estimant victimes de discrimination, de responsables communautaires, d'agents du culte, nous nous efforçons de voir par-delà et en deçà des formes, des idées, des institutions et des pratiques religieuses, pour y découvrir un ensemble complexe d'interactions sociales individuelles et collectives. Derrière le fait religieux sont présents des individus, des acteurs sociaux inégalement dotés en ressources sociales et symboliques, en situation de concurrence, de compétition, de conflit ou de communion, et des types d'organisation ou de structuration communautaire plus ou moins élaborées, selon des modèles plus ou moins centralisés. Cet univers de relations complexes est aussi un espace au sein duquel s'exercent des relations de domination, de lutte, se structurent des organisations sociales et des institutions verticales ou horizontales voire des réseaux sans oublier au passage la présence de dispositifs de contrôle social, d'encadrement des populations ainsi que des formes de résistance qui peuvent prendre appui sur des considérations et user d'un vocabulaire et de thématiques tirés de l'univers religieux, sans nécessairement devoir être regardées et analysées comme motivées par des considérations exclusivement religieuses.

\section{Les conditions sociales d'émergence des mobilisations de musulmans}

La sociologie de l'action collective des minorités religieuses demeure un champ émergent des sciences sociales. Il nous semble néanmoins que les outils de la sociologie des mobilisations s'avèrent utiles pour analyser les formes d'engagement des musulmans dans l'espace public. Plusieurs facteurs sont classiquement mis en avant, relevant des ressources individuelles et collectives, de la structure des opportunités politiques et discursives et des cadres de mobilisation. Un des enjeux ici est d'interroger un paradoxe apparent: alors que les sciences sociales ont de longue date indiqué que la religion - par le type de pratiques sociales qu'elle génère - peut contribuer à l'engagement politique (par la construction d'un capital social, par le type de rappels à l'ordre civiques et politiques dispensés par les institutions religieuses, etc.) il semblerait que les mobilisations de musulmans demeurent un phénomène relativement rare en contextes minoritaires ${ }^{58}$. Deux résultats semblent largement partagés - quelles que soient les approches méthodologiques adoptées - à savoir que les mobilisations de musulmans sont redevables des mêmes facteurs que les autres

${ }^{58}$ Nielsen (J.), dir., Muslim political participation in Europe, op. cit.; Cinalli (M.), Giugni (M.) "Political opportunities, citizenship models and political claim-making over Islam”, Ethnicities, 2013, 13(2), p. 147164. 
formes d'actions collectives (ressources des acteurs, cadrages de la mobilisation, structure des opportunités politiques, etc.), mais qu'elles demeurent relativement marginales dans l'espace des mouvements sociaux en Europe (en termes de fréquence, de nombre d'acteurs mobilisés ou de leurs conséquences politiques) ${ }^{59}$. La plupart des travaux cherchent dès lors à interroger les raisons de cette faiblesse structurelle des mobilisations de musulmans, rapportée en général à la spécificité de la condition minoritaire.

\section{La faiblesse des ressources individuelles et organisationnelles}

Un premier élément systématiquement mis en avant est la faiblesse des ressources des acteurs. En Europe, les individus de confession musulmane, quelle que soit leur façon de vivre leur foi, sont majoritairement des descendants d'immigrés, membres des catégories populaires. Or la sociologie politique a montré de longue date que plus on descend dans la hiérarchie sociale, plus la participation politique est limitée ${ }^{60}$. Quand bien même la force du nombre peut favoriser le passage à l'action collective, les faibles propensions à l'engagement, la défiance à l'égard du politique parmi certaines fractions des classes populaires et le peu de ressources matérielles et politiques ne favorisent pas les mobilisations de musulmans ${ }^{61}$. Ces éléments sont renforcés par la situation juridique dans laquelle se trouve une partie des fidèles, les étrangers, qui disposent de peu de droits civiques, en particulier du droit de vote en France et dans de nombreux pays européens, ce qui ne concourt pas à leur engagement et freine la socialisation politique de leurs descendants ${ }^{62}$. La thèse de «l'apathie politique» ("political quiescence ») des immigrés qui serait liée à leur propre socialisation dans des États peu démocratiques et donc à un usage plus limité des droits politiques - est cependant aujourd'hui largement remise en cause, grâce notamment à des données plus fines ${ }^{63}$. Sylvain Brouard et Vincent Tiberj ont ainsi montré que si la participation associative était plus faible toutes choses égales par ailleurs chez les immigrés et leurs descendants, tout comme leur participation électorale, en comparaison à la population majoritaire, ils affichent en revanche un intérêt pour la politique française plus important ${ }^{64}$. Ces éléments laisseraient dès lors penser que les bases d'une action collective de musulmans ne sont pas entièrement absentes dans les sociétés européennes.

Une autre hypothèse avancée pour expliquer le caractère particulièrement improbable des mobilisations de musulmans, souligne que la structuration même de l'islam serait peu propice à l'action collective. À distance de toute interprétation culturaliste, la thèse se veut institutionnaliste : le caractère décentralisé, voire fragmenté et sans hiérarchie claire (chez les sunnites en tout cas), de cette religion, ne favoriserait pas des formes d'organisations unitaires. Cette fragmentation serait renforcée par certains clivages nationaux, générationnels et théologiques. Elle apparait particulièrement visible à l'échelle nationale, comme l'indiquent les difficultés de légitimation du Conseil français du culte musulman

\footnotetext{
${ }^{59}$ Cette faiblesse tient peut-être également au fait que peu d'organisations non-musulmanes se mobilisent pour des causes liées à l'islam (lutte contre l'islamophobie, etc.). Cinalli et Giugni notent ainsi que les organisations anti-racistes traditionnelles (comme SOS Racisme, le MRAP, la LICRA, la LDH en France) se mobilisent peu - jusqu'à présent - autour de causes relatives à l'islam.

${ }^{60} \mathrm{Cf}$. Gaxie (D.), Le cens caché, op. cit.

${ }^{61}$ De Galembert (C.), « Cause du voile et lutte pour la parole musulmane légitime », op. cit.

${ }^{62}$ Warner (C. M.), Wenner (M. W.), "Religion and the political organization of Muslims in Europe", Perspectives on Politics, 2006, 4(03), p. 457-479.

${ }^{63} \mathrm{Cf}$. les critiques de M. Martiniello à ce sujet: Martiniello (M.), "Political participation, mobilization and representation of immigrants and their offspring in Europe", Willy Brandt Series of Working Papers in International Migration and Ethnic Relations, Malmö, 2005.

${ }^{64}$ Brouard (S.), Tiberj (V.), Français comme les autres ? op. cit.
} 
(CFCM), ou des autres organisations représentatives dans certains pays européens ${ }^{65}$. Le champ organisationnel islamique apparait dès lors traversé par des conflits entre les différentes figures qui prétendent incarner la bonne version de l'islam ${ }^{66}$, conflits auxquels participent également activement certains experts 'musulmans' auditionnés par les missions parlementaires ${ }^{67}$. Ces clivages sont également perceptibles à l'échelle locale, comme l'indique le chapitre d'Etienne Pingaud dans cet ouvrage. L'auteur ne les renvoie néanmoins pas d'abord à des facteurs théologiques, comme une lecture rapide pourrait le laisser penser, mais au positionnement dans le champ politique local et le type de ressources que ces différents entrepreneurs de cause sont en mesure de revendiquer.

Il faut souligner enfin qu'en France, les associations cultuelles ${ }^{68}$ disposent de peu de ressources matérielles et financières. Outre la question du financement des lieux de culte ou des imams, il est difficile de créer des positions salariées au sein de ces organisations, si ce n'est en ayant recours à des financements privés ou issus de fondations. La laïcité a dans ce cas des conséquences très concrètes, contraignant les conditions matérielles de l'organisation collective. Cette précarité financière touche cependant au-delà des associations directement cultuelles, les collectifs minoritaires recevant peu de subsides publics, étant souvent perçus comme "communautaristes». Quand ils sont soutenus, comme le montre Etienne Pingaud dans son chapitre, c'est souvent au prix d'une vassalisation politique dans le cadre de relation clientélaire avec le pouvoir municipal.

\section{Le rôle de la structure des opportunités politiques et discursives}

Au-delà des ressources individuelles et collectives, des travaux récents indiquent que le degré et les formes de mobilisations dépendent fortement du contexte politique, social et idéologique dans lequel elles prennent place, et donc de la structure des opportunités politiques et discursives ${ }^{69}$. Classiquement, on pourrait faire l'hypothèse que les pays ayant une tradition multiculturelle plus ancrée - incarnée par certains dispositifs légaux visant à la reconnaissance et l'obtention de droits pour les minorités ethniques, régionales ou religieuses - sont plus propices à l'action collective des musulmans. Une recherche qui visait précisément à évaluer les effets de la structure des opportunités politiques et discursives sur la mobilisation des musulmans en Europe a assez largement validé cette hypothèse. À partir d'une analyse du contenu de la presse ("claim analysis»), Manlio Cinalli et Marco Giugni indiquent en effet que les pays les plus inclusifs à l'égard des musulmans (en termes de droits individuels et collectifs, de représentation dans le champ politique, etc.) s'avèrent les plus propices à l'expression de revendications (prises de position publiques, manifestations, pétitions, etc.) de la part de mouvements sociaux, de groupes d'intérêts et

${ }^{65}$ Laurence (J.) The emancipation of Europe's Muslims: the state's role in minority integration, Princeton, Princeton University Press, 2011.

${ }^{66}$ De Galembert (C.), « Cause du voile et lutte pour la parole musulmane légitime », op. cit.

${ }^{67}$ Frégosi F, «L'envers de la législation sur les voiles : une domestication de l'islam par la loi », in Revue $d u$ Droit des Religions, novembre 2016, n² 2 p 83-106.

${ }^{68}$ La majorité des associations gérant des salles de prière musulmanes ayant une vocation plus large sont régies par la loi de 1901 et non celle de 1905 (voir par exemple chapitres 1 et 5).

${ }^{69} \mathrm{Par}$ structure des opportunités discursives on entend les normes qui régissent la prise de parole dans l'espace public. Celles-ci peuvent en effet être plus ou moins ouvertes à l'expression du désaccord et de la diversité de points de vue. Ces normes influent sur la capacité des revendications et des identités collectives à accéder à la visibilité dans les médias, de raisonner avec d'autres demandes d'acteurs collectifs et de parvenir à une certaine légitimité dans l'espace public. Cf. Koopmans (R.), Statham (P.), Giugni (M.), Florence (F.), Contested Citizenship: Immigration and Ethnic Relations Politics in Europe. Minneapolis: Minnesota University Press, 2005. 
d'associations $^{70}$. La Grande-Bretagne apparait comme le pays le plus ouvert à cet égard, la Suisse et l'Allemagne semblent à l'inverse moins propices à l'organisation collective des musulmans, alors que la France et les Pays-Bas se situent dans une position intermédiaire. La variable décisive pour comprendre les mobilisations de musulmans serait dès lors le contexte politique (national). Le chapitre de Corinne Torrekens dans cet ouvrage indique ainsi que l'investissement du champ politique par les musulmans à Bruxelles - que ce soit par la création de partis politiques autonomes, la participation à certaines listes électorales ou des formes de lobbying - dépend aussi du contexte national, de telles mobilisations apparaissant particulièrement marginales en France par exemple. Les différences sont cependant moins importantes qu'on aurait pu l'imaginer, les mobilisations se situant à un niveau assez proche en France et en Grande-Bretagne, en dépit de leurs traditions différentes de gestion des minorités ${ }^{71}$.

Ces recherches, aussi sophistiquées soient-elles, reposent principalement sur des méthodes quantitatives et l'analyse de la presse, et ne peuvent saisir les variations locales et les conditions pratiques de mobilisation des musulmans. La prise en compte du contexte politique, institutionnel et discursif requiert également de porter le regard sur des formes plus localisées d'engagement, et ce d'autant plus qu'en France les questions et revendications soulevées par les groupes musulmans concernent avant tout les administrations locales et en particulier municipales ${ }^{72}$. Ces mobilisations risquent dès lors de passer sous le radar des recherches quantitatives, qui ne s'intéressent qu'aux mouvements nationaux ou disposant d'un niveau de visibilité suffisant pour accéder à la presse nationale ${ }^{73}$. C'est tout l'enjeu de ce livre, qui s'intéresse principalement à des mobilisations locales de musulmans.

\section{Des motifs et des formes d'engagements qui dépendent de la position sociale}

Les recherches qui mettent l'accent sur le rôle de la structure des opportunités discursives - qui s'intéressent dès lors à la façon dont il est possible de s'exprimer et de faire valoir des revendications dans l'espace public - invitent à s'intéresser aux processus de cadrage des mobilisations de musulmans. À la question « Au nom de quoi s'engagent-ils? ", trois types de motifs peuvent être distingués ${ }^{74}$. Tout d'abord, les musulmans ayant plusieurs

${ }^{70}$ Cinalli (M.), Giugni (M.) "Political opportunities, citizenship models and political claim-making over Islam", Ethnicities, 2013, 13(2), p. 147-164.

${ }^{71}$ Gianni (M.), Giugni (M.G.), "Resources, Opportunities, and Discourses: What Explains the Political Mobilization of Muslims in Europe?", in Chabannet (D.), Royall (F.), dir., From Silence to Protest: International Perspectives on Weakly Resourced Groups, Farnham, Ashgate, 2014, p. 113-118. Les travaux de Valérie Sala Pala, qui portent cependant non sur des mobilisations de musulmans mais de minorités ethniques sur la question du logement, parviennent à des résultats tout aussi contre-intuitifs "Alors que tout semble les opposer, les stratégies différentialiste et universaliste observées à Birmingham et à Marseille posent un même problème: elles tendent à invisibiliser (ou minimiser) le poids des discriminations dans l'expérience des minoritaires et dans la production des inégalités ethniques; ce faisant elles conduisent à la marginalisation de la lutte contre les discriminations. (...) Ces deux lectures tendent in fine à imputer l'expérience des minoritaires à leurs caractéristiques propres plus qu'à la construction d'un ordre social ethnicisé, qui classe chaque individu en fonction de ses origines ». Cf. Sala Pala (V.), Discriminations ethniques. Les politiques du logement social en France et au Royaume-Uni, Rennes : Presses Universitaires de Rennes, 2012.

${ }^{72}$ De Galembert (C.), «Une action publique en quête de normes : la gestion publique de l'islam », in Le Bart (C.), Pasquier (R.), Arnaud (L.), dir., Idéologies et action publique territoriale : La politique change-t-elle encore les politiques?, Rennes, Presses universitaires de Rennes, 2007.

${ }^{73} \mathrm{Le}$ Bohec (J.), «À propos de l'utilisation de la presse comme source de données ». L'aquarium, 1991.

${ }^{74}$ Nous reprenons ici les développements de Frégosi (F.), «Formes de mobilisation collective des musulmans en France et en Europe », op. cit. 
identités à disposition - par exemple celles d'étudiants en ascension sociale, de travailleurs précaires ou sans-papiers, de descendants d'immigrés, de minorités racisées, etc. - peuvent s'engager en faveur de causes très variées, qui n'ont que peu à voir avec la religion ${ }^{75}$. On trouve ensuite les mobilisations qui ont pour visée certaines revendications proprement religieuses ou cultuelles : constructions d'une mosquée ou d'un carré musulman dans un cimetière, respect des interdits alimentaires, etc. Enfin, les musulmans peuvent se mobiliser contre les discriminations ou la stigmatisation dont ils font l'objet, et donc pour l'égalité de traitement, le cadrage dominant relevant désormais de la lutte contre l'islamophobie. Le chapitre d'Houda Asal indique d'ailleurs la constitution en cours d'un champ national et peut-être européen de la lutte contre l'islamophobie, où les acteurs principaux, en France, sont le Collectif contre l'islamophobie en France (CCIF), le Collectif contre le racisme et l'islamophobie (CRI) et le Parti des Indigènes de la République - qui oscillent entre concurrence et coopération, au gré des considérations stratégiques et des positionnements politiques, sans oublier l'Observatoire national de lutte contre l'islamophobie rattaché au CFCM.

Si les travaux manquent à ce sujet, il peut être intéressant d'étudier plus finement la sociologie de ces différentes formes d'action collective. Nagel et Straehei indiquent ainsi, dans le cas britannique, que les formes d'engagement varient fortement selon la position dans la hiérarchie sociale et ethnique. Les "arabes musulmans", plus proches des classes moyennes, mettent sous l'éteignoir leur appartenance religieuse qui les rapprocherait des " musulmans sud-asiatiques » (pakistanais, indiens, bengalis ou indonésiens), membres des fractions les plus précarisées du sous-prolétariat, dont ils cherchent à se démarquer. Le cadrage des mobilisations est régi par des stratégies de distinctions, qui font que les musulmans originaires d'Asie du Sud se mobilisent souvent en tant que musulmans et au nom de l'islam, quand les Arabes s'engagent pour des causes plus larges, leur montée en généralité étant facilitée par leurs dispositions sociales ${ }^{76}$. On retrouve des phénomènes similaires aux Pays-Bas, où les luttes de Marocains et de Turcs sont cadrées différemment 77 . Les premiers se mobilisent davantage autour de revendications religieuses, quand les seconds s'engagent autour de causes plus générales et politiques. Outre le positionnement dans la hiérarchie sociale, les auteurs adoptent ici une perspective relationnelle, mettant en évidence le rôle de l'expérience de la discrimination et de la stigmatisation dans ces stratégies différenciées de mobilisation.

\footnotetext{
${ }^{75} \mathrm{Nagel}$ et Staeheli indiquent ainsi que la majorité des militants britanniques qu'ils ont interviewé ne se mobilisent pas sur une base religieuse. Cf. Nagel (C. R.), Staeheli (L. A.), "Muslim political activism or political activism by Muslims? Secular and religious identities amongst Muslim Arab activists in the United States and United Kingdom". Identities, 2013, 18(5), p. 437-458.

${ }^{76}$ Pour des résultats similaires, remontant aux années 1990, cf. Statham (P.), "Political Mobilisation By Minorities In Britain: A Negative Feedback Of 'Race Relations'?", Journal of Ethnic and Migration Studies, 25 (4), p. 597-626, 1999.

${ }^{77}$ Cf. Phalet (K.), Baysu (G.), Verkuyten (M.) "Political mobilization of Dutch Muslims: Religious identity salience, goal framing, and normative constraints", Journal of Social Issues, 2010, 66(4), p. 759-779. À noter qu'on manque significativement d'éléments de ce point de vue dans le cas français. La seule forme de participation politique pour laquelle on dispose de données relatives aux effets de la position sociale est la pratique électorale. Le rapport au vote des musulmans français apparait alors fortement influencé par leur place dans la hiérarchie sociale et éventuellement territoriale. On sait en revanche peu de choses quant au rapport différencié au politique de différentes fractions des communautés musulmanes. Est-ce que les musulmans descendants de turcs votent ou s'engagent de la même façon que les musulmans descendants d'Algériens? Cf. néanmoins Simon (P.), Tiberj (V.), «La fabrique du citoyen: origines et rapport au politique en France », in Beauchemin (C.), Hamel (C.), Simon (P.), dir., Trajectoires et origines. op. cit., p. 501-529.
} 
Si on manque de données dans le cas français, on sait néanmoins que davantage que la position sociale en tant que telle, c'est la trajectoire qui importe. Leyla Arslan avance ainsi à partir d'une série d'entretiens biographiques que les jeunes qui connaissent une trajectoire d'ascension sociale rejettent les formes d'organisations collectives communautaires, ne voulant être réduits à leur identité ethnique ou religieuse ${ }^{78}$. À l'inverse, comme l'indique Camille Hamidi, les trajectoires de relégation sociale semblent davantage conduire à mettre en avant une identité ethnique, territoriale ou religieuse spécifique et à faire de la discrimination un élément structurant du rapport au monde social ${ }^{79}$. Le chapitre de Nathalie Fuchs dans ce livre complexifie ces analyses: les mobilisations associatives qu'elle étudie sont nées en effet d'acteurs en voie d'ascension sociale qui ne rejettent pas le référent religieux.

\section{L'engagement comme réaction à l'expérience des discriminations ?}

Une dernière hypothèse visant à expliquer les conditions de mobilisation des musulmans en situation minoritaire mérite d'être explorée : elle souligne les effets de la stigmatisation et de la discrimination sur le rapport au politique. S'il existe à ce jour peu de travaux adoptant une telle perspective à propos de l'engagement des croyants, certains éléments tirés de recherches sur les conséquences de l'expérience minoritaire de certains groupes ethno-raciaux sont éclairants.

\section{De la ségrégation à la conscience minoritaire}

Il existe en effet une littérature anglo-saxonne foisonnante sur la participation et le comportement politique (principalement électoral) des minorités ethno-raciales. Un des enjeux des travaux américains de Rodney Hero ${ }^{80}$, Reuel Rogers ${ }^{81}$ ou de Michael Dawson ${ }^{82}$ est d'expliquer le comportement électoral spécifique des Africains-Américains et des Latinos, qui votent de façon disproportionnée en faveur des démocrates. Deux mécanismes explicatifs ont été mis en avant, qui soulignent tous les deux la relation entre visibilité et politisation, ce que Hero qualifie de «two-tiered pluralism ». D'une part, ce qui distingue les Africains-Américains et les Latinos des autres groupes raciaux aux Etats-Unis, c'est leur couleur de peau, qui les rend visibles et donc sujets potentiels à stigmatisation ou discrimination (davantage que les immigrés blancs par exemple). L'orientation à gauche de ces minorités serait donc liée à leur «visibilité» et à l'expérience qu'ils font de la discrimination, de l'altération, du fait d'être perçus comme "autres» ou "différents ». D'autre part, cet alignement politique particulier pourrait s'expliquer par la conscience spécifique que font naitre ces expériences discriminatoires, qu'ils soient directement touchés par les préjugés ou non. Michael Dawson qualifie ce phénomène de "destin lié » avec le reste du groupe, c'est à dire le sentiment d'appartenir à un groupe partageant les mêmes problèmes (et auquel on va majoritairement donner les mêmes réponses). Comme le concluent Vincent Tiberj et Patrick Simon : «La conjonction de ces deux mécanismes les pousse à soutenir le parti (ou le camp) politique qui leur semble le mieux à même de les défendre face aux discriminations qu'eux-mêmes ou leurs proches peuvent subir. Ce n'est

\footnotetext{
${ }^{78}$ Arslan (L.), Enfants d'islam et de Marianne. Des banlieues à l'université, Paris, PUF, 2010.

${ }^{79}$ Hamidi (C.), « Catégorisations ethniques ordinaires et rapport au politique », Revue Française de Science Politique, 60(4), 2010.

${ }^{80}$ Hero (R.), Latinos and the US Political System. Two Tiered Pluralism, Philadelphia, Temple University Press, 1992.

${ }^{81}$ Chong (D.), Rogers (R.), "Racial solidarity and political participation", Political Behavior, 2005, 27(4), p. 347-374.

82Dawson (M.) Behind the Mule, Princeton, Princeton University Press, 1994.
} 
donc pas la visibilité en tant que telle qui amène à la gauche, c'est la réaction de la société à cette visibilité83. »

Ces deux auteurs ont tenté d'appliquer ce modèle explicatif pour analyser le vote massivement à gauche des descendants d'immigrés et des musulmans dans le cas français ${ }^{84}$. À partir des données de l'enquête «Trajectoires et Origines » ils démontrent que le positionnement à gauche des minorités en France n'est en rien lié à certaines spécificités culturelles mais est fortement corrélé à une variable : le fait «d'être vu comme Français ». Toutes choses égales par ailleurs, moins les individus sont perçus comme Français, alors qu'ils le sont, plus ils ont de propension à voter à gauche. Ce segment de l'offre politique est vu comme le plus à même de prendre en charge leur discrimination vécue ou ressentie. L'altérisation des groupes minorisés produit donc des comportements politiques spécifiques.

Qu'en est-il des comportements politiques non-électoraux et éventuellement protestataires? Hechter et Okamoto, dans une revue de la littérature internationale sur ces questions, mettent en avant deux facteurs principaux pour expliquer l'action collective des groupes minorisés ${ }^{85}$. Tout d'abord, la formation d'une identité collective apparait essentielle, ce qui s'avère particulièrement ardu pour des groupes historiquement stigmatisés, dont les membres pourraient avoir tendance à se détourner. À ce titre, on peut noter qu'il existe peu à ce jour - bien qu'on manque de recherches à ce sujet - de mouvements de type "muslim pride», comme il a existé des luttes symboliques défendant le «black is beautiful» ou la "gay pride». Second élément, au-delà de la conscience d'appartenir à un groupe discriminé, l'existence d'organisations collectives est nécessaire pour articuler une série de revendications, sanctionner les comportements de free riding et plus largement façonner le groupe. Toute une littérature sur la participation des minorités ethno-raciales en Europe s'intéresse dès lors aux facteurs permettant d'expliquer le caractère très variable de la densité organisationnelle et de l'offre politique selon les groupes ethniques. Comment expliquer que certaines communautés soient plus organisées que les autres? La plupart des recherches soulignent que la force des liens communautaires dépend du degré de discriminations subies. Maxwell a ainsi montré, dans le cas britannique, que les minorités issues de la péninsule indienne, plus discriminées sur les marchés du travail et du logement que les Afro-Caribéens, s'appuient davantage que ces derniers sur les réseaux communautaires pour trouver un emploi ou un appartement, ce qui se traduit par des réseaux interpersonnels plus denses et des formes d'organisation collective plus solides $^{86}$. La concentration territoriale résultant de la ségrégation se traduirait par des réseaux communautaires denses - un capital social élevé pour reprendre le prisme très putnamien de ces recherches - qui favoriseraient l'organisation collective et l'émergence d'un sentiment d'appartenance au groupe, se traduisant par des actions collectives plus

${ }^{83}$ Tiberj (V.), Simon (P.), « La fabrique du citoyen » op. cit.

${ }^{84}$ Les Français de confession musulmane ont ainsi très majoritairement voté en faveur de François Hollande au Second tour des elections présidentielles de 2012.

${ }^{85}$ Hechter (M.), Okamoto (H.), "The Political Consequences Of Minority Group Formation", Annual review of political science, 2001.

${ }^{86}$ Maxwell (R.), Ethnic Minority Migrants in Britain and France: Integration Trade-Offs, Cambridge University Press: New York, 2012. Pour des résultats relativement similaires sur les Pays-Bas, voir Fennema (M.), Tillie (J.) "Political participation and political trust in Amsterdam: civic communities and ethnic networks", Journal of ethnic and migration studies, 1999, 25(4), p. 703-726 ; Tillie (J.) "Social capital of organisations and their members: explaining the political integration of immigrants in Amsterdam", Journal of Ethnic and Migration Studies, 2004, 30(3), 529-541; sur le Danemark cf. Togeby (L.) "It depends... how organisational participation affects political participation and social trust among second-generation immigrants in Denmark", Journal of Ethnic and Migration Studies, 2004, 30(3), p. 509528. 
fréquentes (que pour les groupes moins soudés) et des comportements électoraux spécifiques. Le groupe viendrait dès lors compenser les ressources individuelles limitées des acteurs pour expliquer des mobilisations sinon improbables du moins relativement rares. On retrouve ici la thèse du ghetto protecteur pour les minorités ${ }^{87}$ : la ségrégation sociale et résidentielle serait vectrice de conscience et d'engagement politique ${ }^{88}$. Dans la même veine, Claudine Gay a démontré qu'aux Etats-Unis les politiques de mixité sociale, et donc la dispersion spatiale des minorités ethno-raciales, s'étaient traduites par un déclin de leur participation politique ${ }^{89}$.

Ces travaux portent cependant principalement sur les minorités ethno-raciales, et non sur des minorités religieuses. Or, la transivité entre l'ethnicité et la religion ne va évidemment pas de soi. Non seulement les musulmans ne représentent qu'une partie des membres des groupes minorisés - même parmi les descendants français d'immigrés originaires de Turquie, du Maghreb d'Afrique sub-saharienne, seuls 59\% se déclarent musulmans au milieu des années $2000^{90}$ - mais l'affiliation religieuse s'avère moins décisive que l'origine (et donc la couleur de peau en concluent Tiberj et Michon) dans l'orientation à gauche des groupes minorisés concernés ${ }^{91}$. Ici encore cependant quelques études qualitatives permettent d'affiner ces conclusions en les élargissant à d'autres formes de participation. Romain Garbaye, dans son étude des rapports entre municipalités et associations locales en Grande-Bretagne, indique que c'est précisément la non prise en compte des revendications religieuses de ces dernières qui les ont conduit sur la voie de la protestation et de l'organisation collective, la fermeture des opportunités politiques locales - vécue comme une discrimination, les autres minorités religieuses étant traitées avec plus d'égard à leurs yeux - se traduisant par une radicalisation des modes d'action ${ }^{92}$. Des processus similaires ont été observés à Strasbourg par Claire Donnet ${ }^{93}$. Ici aussi c'est une expérience vécue comme discriminatoire - le refus du maire de céder un terrain pour la construction d'une nouvelle mosquée - qui est à l'origine de la trajectoire de politisation de l'association, ou de certains de ses membres, et de son usage d'un répertoire d'action plus protestataire (manifestations, pétitions, etc.). L'infléchissement se fait également sentir d'un point de vue discursif, l'association devenant le héraut d'un discours égalitaire dans l'espace public. À l'image du chapitre de Nathalie Fuchs sur le Val Fourré dans cet ouvrage, religion et action collective semblent marcher main dans la main dans le cas strasbourgeois: " l'inégalité religieuse en matière de culte est la pierre d'achoppement sur laquelle ils font reposer leur revendication de justice sociale ${ }^{94}$. »

${ }^{87}$ Voir St. Clair Drake et al., Black Metropolis: A Study of Negro Life in a Northern City, Chicago, The University of Chicago Press, 1943. Pour la France, Lapeyronnie (D.), Ghetto urbain : ségrégation, violence, pauvreté en France aujourd'hui, Paris, Robert Laffont, 2008.

${ }^{88}$ Maxwell (R.) "Political participation in France among non-European-origin migrants: Segregation or integration?", Journal of Ethnic and Migration Studies, 2010, 36(3), p. 425-443.

${ }^{89}$ Voir Gay (C.), "Spirals of Trust? The Effect of Descriptive Representation on the Relationship Between Citizens and Their Government.", American Journal of Political Science, 2002, 46(4), p. 717-32.

${ }^{90}$ Selon l'enquête RAPFI, cf. Brouard (S.), Tiberj (V.), Français comme les autres ?, op. cit.

${ }^{91}$ Voir Tiberj (V.), Michon (L.) "Two-tier pluralism in 'colour-blind' France”, West European Politics, 2013, 36(3), p. 580-596.

${ }^{92}$ Garbaye (R.), Getting into Local Power: The Politics of Ethnic Minorities in British and French Cities, Oxford, Blackwell. 2005. Voir également Garbaye (R.), «Les organisations musulmanes et l'État britannique, de 1980 à 2005 : du local au national », Hérodote, 2010, (2), p. 57-69.

${ }^{93}$ Donnet, (C.) «Une mosquée pour exister», op. cit. Pour des processus relativement similaires pour la Suisse, cf. Mesgaezadesh (S.), Nedjar (S.), Bennani-Chraïbi (M.), " "L'organisation" des musulmans de Suisse. Dynamiques endogènes et injonctions de la société majoritaire », in Monnot (C.), dir., La Suisse des mosquées, op. cit, p. 53-77.

${ }^{94}$ Ibid., p. 220. 


\section{Une approche relationnelle de la politisation de l'expérience des discriminations}

Il convient cependant d'éviter toute perspective mécaniste qui ferait de l'expérience de la discrimination ou de la concentration spatiale le ferment systématique de la mobilisation collective, sans quoi on ne pourrait comprendre le caractère relativement limité des mobilisations de musulmans en Europe. Ici encore la perspective relationnelle s'avère fructueuse. Statham, comparant les groupes sud-asiatiques et afro-caribéens en GrandeBretagne, explique ainsi les mobilisations plus importantes des premiers par la moins forte cooptation de ses élites par le système politique ${ }^{95}$, qui en fait des agents de mobilisation de leur communauté plus importants. Garbaye montre également comment, pour une période plus récente, des relations clientélistes se sont établies entre associations musulmanes et certaines municipalités travaillistes. L'accès à certaines demandes concernant les interdits alimentaires dans les cantines scolaires ou la pratique du sport à l'école pour les filles constitue un moyen de s'assurer une clientèle électorale stable pour la gauche britannique, se traduisant alors par un déclin des formes d'action plus revendicatives ${ }^{96}$.

$\mathrm{Si}$ des recherches sur les formes d'action collective des musulmans commencent à émerger - de façon plus récente en France - l'analyse du rôle de l'expérience des discriminations et de la stigmatisation dans ces processus demeure un chantier ouvert. Il conviendrait en particulier de creuser la question du passage à l'action collective des individus discriminés. À ce titre, il faudrait s'intéresser non seulement aux formes de mobilisations de musulmans - du côté de l'offre d'engagement - mais aussi à la demande, c'est à dire au rapport ordinaire aux discriminations liées à l'islam. Comment les discriminations sont-elles vécues? Sont-elles ou non politisées? Par qui ? Dans quel contexte? Le chapitre de Julien Beaugé s'avère extrêmement riche à cet égard. À partir de l'étude de la trajectoire d'une lycéenne qui a vécu son dévoilement après la loi de 2004 comme une discrimination, il montre le déploiement de formes de résistance à bas-bruit, une politisation fugace, puis le repli sur la sphère privée. Il indique que l'offre associative et politique, pour prendre en charge l'expérience discriminatoire, s'est avérée limitée ou inadéquate, ne favorisant pas la mobilisation. Plus largement, la thèse de Julien Beaugé indique comment les femmes voilées qu'il a interviewées ne politisent que très rarement leur expérience, en dépit de la stigmatisation dont elles sont l'objet et de la souffrance qu'elles endurent ${ }^{97}$. Au-delà de leurs dispositions peu propices à la politisation, il indique comment les épreuves que sont le port du voile ou le dévoilement, sont tues (et donc impossible à publiciser et politiser), relativisées ou rationalisées religieusement. La discrimination est donc loin de conduire automatiquement à l'engagement.

Il semble également que les interprétations données à ces discriminations et les façons d'y réagir dépendent aussi de certains facteurs proprement religieux. L'audience croissante du salafisme dans les quartiers populaires européens, peut être lue comme une réponse à la stigmatisation ${ }^{98}$. Ce courant défend en effet une conception quiétiste de l'islam, principalement apolitique ${ }^{99}$, voire antipolitique. Le politique et toute forme d'engagement y compris contre l'islamophobie, comme l'indique Eric Marlière dans son chapitre ici - sont rejetés afin de préserver la croyance de toute intrusion de l'État. Fareen Parvez, qui a effectué une étude ethnographique dans une mosquée salafie en banlieue lyonnaise, indique ainsi que l'ostracisation a bien pour effet de renforcer la communauté morale, mais la lutte

\footnotetext{
${ }^{95}$ Statham (P.), "Political Mobilisation By Minorities In Britain: A Negative Feedback Of 'Race Relations'?”, op. cit.

${ }^{96}$ Garbaye (R.), Getting into Local Power, op. cit.

${ }^{97}$ Beaugé (J.), La force d'une institution disqualifiée : les logiques sociales du voilement des musulmanes en France, Thèse pour le doctorat de science politique, Université d'Amiens, 2013.

${ }^{98}$ Amghar (S.), L'islam militant en Europe, Paris, Infolio, 2013.

${ }^{99}$ Adraoui (M.-A.), Le salafisme : du golfe aux banlieues, Paris, PUF, 2013.
} 
qui en découle vise à défendre la sphère privée contre les incursions intrusives de l'État, non à s'engager collectivement ${ }^{100}$.

C'est ainsi toute une gamme de réponses à la stigmatisation qui semble s'esquisser, allant de l'action collective à la rationalisation religieuse de la souffrance vécue et à des formes plus sourdes et infra-politiques de résistance. À ce titre, les travaux de Michèle Lamont sur les réponses à la stigmatisation s'avèrent précieux. Elle met en avant la variabilité selon le contexte national, et l'historicité propre à chaque groupe minorisé, pour comprendre pourquoi ces réponses oscillent entre des formes organisées de lutte et des résistances moins visibles ${ }^{101}$. À cet égard, les réactions des Africain-Américains au racisme et à la ségrégation - prenant la forme d'un mouvement social très structuré 102 - loin d'être la règle, s'avèrent assez uniques.

On peut dès lors esquisser une hypothèse, au sujet des musulmans, permettant de rendre compte de la faiblesse des mobilisations en Europe ou, pour reformuler la question de façon plus précise, de comprendre pourquoi les entreprises de mobilisation des musulmans - qui sont nombreuses, comme en attestent les différents chapitres de cet ouvrage et la littérature présentée ici - ne rencontrent qu'un écho limité parmi les publics qu'elles ciblent. Ce phénomène relève peut-être d'un décalage d'ordre sociologique entre les entrepreneurs de cause musulmans et leurs publics. D'un côté, les courants les mieux implantés dans les milieux populaires, comme le Tabligh et plus récemment le salafisme, développent une conception quiétiste et dépolitisée de l'islam, n'incitant pas à l'engagement et plutôt au retrait ascétique. De l'autre, ceux qui cherchent à encourager une « citoyenneté islamique » (l'UOIF, le Millî Görüs, etc.) sont les organisations les plus élitistes dans leur recrutement, qui touchent assez peu les classes populaires en Europe. Ce décalage est renforcé par le fait que les élites musulmanes, en voie d'ascension sociale, potentiellement les plus disposées à l'engagement, sont les moins à même à endosser cette identité collective perçue comme stigmatisante. Si ce décalage peut être variable selon les pays et les contextes politiques, il apparait comme un élément structurant -- et à ce jour peu relevé103 - permettant de comprendre pourquoi les mobilisations de musulmans demeurent jusqu'à présent si marginales.

\section{Comment se mobilise-t-on au nom de l'islam ?}

Si les mobilisations de musulmans sont rares, c'est surtout qu'elles prennent des formes peu visibles, voire non-publicisées, s'enracinant en outre principalement à l'échelle locale. Les quelques recherches quantitatives indiquent que l'expression des revendications - peu distinctes selon les pays - prend le plus souvent des formes policées et conventionnelles, l'action collective non-conventionnelle (manifestations, occupations, désobéissance civile, etc.) demeurant exceptionnelle, et moins importante que pour d'autres secteurs des mouvements sociaux ${ }^{104}$. Alors que la construction de mosquées fait l'objet de conflits

\footnotetext{
${ }^{100}$ Parvez (Z. F.), "Debating the burqa in France: The antipolitics of Islamic revival", Qualitative Sociology, 2011, 34(2), p. 287-312.

${ }^{101}$ Lamont (M.), Mizrachi (N.), dir., "Introduction: Ordinary People Doing Extraordinary Things: Responses to Stigmatization in Comparative Perspective". Ethnic and Racial Studies, 35(3), 2012, p. 365-381.

${ }^{102}$ Cf. McAdam (D.), Political process and the development of black insurgency, 1930-1970, Chicago, University of Chicago Press, 1980 ; Morris (A.), Origins of the Civil Rights Movement, op. cit.

103Voir néanmoins Amghar (S.), L'islam militant en Europe, op. cit.

${ }^{104}$ Cinalli (M.), Giugni (M.), "Political opportunities, citizenship models and political claim-making over Islam", op. cit. ; Statham (P.), "Political mobilisation by minorities in Britain: Negative feedback of 'race relations'?", Journal of Ethnic and Migration Studies, 1999, 25(4), p. 597-626.
} 
importants dans les pays occidentaux depuis les années $1980^{105}$, variables selon les pays et les configurations locales, rares sont les manifestations revendiquant publiquement le droit à des conditions dignes d'exercice du culte. Indépendamment des réactions parfois violentes des opposants (de riverains ou de certains mouvements d'extrême-droite), les formes de mobilisation des associations musulmanes semblent surtout dépendre du degré d'ouverture de la municipalité ${ }^{106}$. Ainsi, la fin de non-recevoir donnée par le maire de Strasbourg se traduit par une mobilisation accrue de l'association porteuse du projet et une dénonciation virulente de la municipalité107. En Italie, où les mobilisations islamophobes relatives à la construction de mosquées sont fréquentes (à l'initiative notamment de la Ligue du Nord), les organisations musulmanes semblent éviter le choc frontal, préférant aux manifestations les accommodements et les négociations discrètes avec les élus ${ }^{108}$. Quand les édiles s'avèrent conciliants les musulmans se font discrets, ne réagissant pas aux attaques dont ils peuvent faire l'objet. Ici, l'action collective se fait à bas-bruit, par le recueil de fonds, éventuellement des pétitions ou la recherche d'alliés parmi les autres groupes religieux.

Les « affaires du voile » ont en revanche débouché sur des manifestations et des actions collectives protestataires en France. Si les premières controverses, en 1989, n'avaient donné lieu qu'à des mobilisations limitées - le contexte des manifestations liées à l'Affaire Rushdie quelques mois auparavant n'incitant pas la majorité des organisations musulmanes à se mobiliser - la résurgence de la question du voile en 2003-2004 a donné lieu à des actions beaucoup plus importantes. On pense notamment à la manifestation de février 2004, organisée par le collectif Une école pour tous-tes qui est parvenu à rassembler des acteurs extrêmement hétérogènes, des organisations proches de Tarik Ramadan (Collectif des musulmans de France, Présence Musulmane, etc.), des militants des quartiers populaires (Mouvement immigration banlieue, Saïd Bouamama), des associations anti-racistes (MRAP, LDH), certains partis politiques de gauche (Verts, LCR) et des collectifs féministes autour de Christine Delphy ${ }^{109}$. C'est précisément cette coalition d'acteurs, dont certains rompus à l'action collective, qui a permis l'émergence de cette protestation. Ici tout le répertoire d'action protestataire a été mobilisé : pétitions, meetings, manifestations, tribunes dans la presse, etc. L'apport de ces acteurs était d'autant plus important que certaines organisations représentatives - et en particulier l'Union des organisations islamiques de France (UOIF) ne pouvait jouer ce rôle de coordination du fait de leur notabilisation associée à la création du CFCM et une position de coopération avec le gouvernement ${ }^{110}$.

Cette alliance n'a cependant duré qu'un temps, les organisations antiracistes et les partis politiques prenant leurs distances une fois la mobilisation retombée. De nouvelles organisations ont depuis vu le jour - CCIF, CRI, MIR - élargissant leurs revendications audelà de la question du voile, pour s'attaquer plus largement à l'islamophobie, reprenant à leur compte l'émergence du référentiel des discriminations. Cette autonomisation de

${ }^{105}$ Césari (J.), "Mosque conflicts in European cities", Journal of Ethnic and Migration Studies, 2005, 31(6), p. 1015-1024; Allievi (S.), dir., Mosques in Europe. Why a solution has become a problem, Londres, alliance Publishing Trust, 2010.

${ }^{106}$ De Galembert (C.), «Une action publique en quête de normes », op. cit.

${ }^{107}$ Donnet, (C.) «Une mosquée pour exister », op. cit.

${ }^{108}$ Saint-Blancat (C.), Schmidt Di Friedberg (O.), "Why are Mosques a Problem ? Local Politics and Fear of Islam in Northern italy", Journal of ethnic and Migration Studies, 31 (6), 2005, p. 1083-1104; sur un processus participatif autour de la construction d'une mosquée à Florence cf. O'Miel J., Talpin J., «Espace et conflits dans la participation. Luttes symboliques et matérialité d'une controverse autour la localisation d'une mosquée à Florence », Lien social et Politiques, 73, 2015, p. 33-52.

${ }^{109}$ De Galembert (C.), «Cause du voile et lutte pour la parole musulmane légitime », op. cit.

${ }^{110} \mathrm{Au}$-delà du cas français, la cooptation de certaines élites musulmanes via la création partout en Europe d'instances représentatives du culte musulman a contribué au déclin de postures plus contestataires depuis les années 1990. Cf. Laurence (J.) The emancipation of Europe's Muslims, op. cit. 
l'espace de la lutte anti-islamophobe peut être lue comme un affaiblissement, celle-ci manquant de soutien et de relais permettant de faire progresser la cause. La quête d'autonomie de cette nouvelle génération militante - très défiante à l'égard de la gauche et de l'extrême-gauche qu'elle voit souvent comme paternaliste et «blanche » - risque ainsi de conduire à sa marginalité, et ce d'autant plus qu'elle se redouble d'importantes divisions internes quant aux stratégies et tactiques à adopter. Cette quête d'autonomie ne peut cependant se comprendre qu'à l'aune du désintérêt des partis gauche pour l'enjeu des discriminations, marqué par des pratiques racistes en interne ou des tentatives de récupération des mouvements sociaux, à l'image de la Marche pour l'égalité et contre le racisme de 1983 et la création de SOS racisme qui en a suivi ${ }^{111}$. L'autonomie qui en découle est à la fois politique et financière, toutes les organisations de lutte contre l'islamophobie étant autofinancées, que cela relève d'un choix ou d'une contrainte liée au refus des collectivités locales de soutenir des actions perçues comme "communautaristes ». Les conditions matérielles des mobilisations de musulmans demeurent dès lors précaires.

Les luttes contre l'islamophobie usent, à ce jour, de deux modes d'action principaux. Elles opèrent tout d'abord un travail d'information et de recensement des actes islamophobes, la connaissance étant perçue comme une arme dans la lutte pour la reconnaissance et pour sortir du déni français autour de ces enjeux. L'information passe également par des campagnes médiatiques dénonçant la stigmatisation des musulmans. Elles mènent ensuite le combat sur le terrain du droit, cherchant à déposer des plaintes pour discrimination ou traitement inégal. Si la judiciarisation des modes d'action s'observe dès la fin des années 1980, la création de la Haute autorité de lutte contre les discriminations et pour l'égalité (HALDE) en 2005 a accéléré ce processus. Cette dernière est ainsi saisie dès sa création par le CCIF au sujet de l'interdiction faite à certaines mères voilées d'accompagner les sorties scolaires. Le nombre de plaintes n'a depuis cessé d'augmenter, favorisé par l'appui d'avocats dédiés. Cette judiciarisation permet également d'ennoblir la cause en la plaçant sur le terrain de la justice. Certaines organisations - comme le MIB autrefois, le Forum Social des Quartiers Populaires, le Parti des Indigènes de la République ou le Front Uni de l'Immigration et des Quartiers Populaires - demeurent dans une posture plus critique, préférant au répertoire juridique des formes d'action plus directes, allant des manifestations aux assises contre l'islamophobie. Ces initiatives plus radicales - mêlant lutte contre l'islamophobie et revendications de justice sociale - peinent cependant à ce jour à trouver leur public. Le chapitre d'Alexandre Piettre indique comment ces initiatives tentent de percoler localement, esquissant les contours de ce qui pourrait être une "gauche islamique ».

Les chapitres qui suivent présentent une série d'études localisées, principalement dans des quartiers populaires. Elles sont le fruit d'enquêtes ethnographiques fouillées, reposant sur l'observation de réunions, de manifestations ou de moments de sociabilité informels et de nombreux entretiens avec des fidèles, des représentants associatifs ou des élus. Certains chapitres adoptent une posture encore plus fine, retraçant le parcours d'engagement ou de retrait de croyants dont la trajectoire est suivie en détail et sur le temps long par les auteurs.

Ces recherches permettent de visibiliser les capacités d'action des minorités musulmanes - ce qu'on qualifie en anglais d'agency - preuve qu'en dépit de la stigmatisation et des discriminations qu'elles subissent, la domination ne s'impose pas à elles unilatéralement, sans provoquer de réactions. Celles-ci sont diverses, pouvant conduire à l'engagement politique ou associatif, ou à l'inverse à des formes de mise à distance du jeu politique, perçu comme corrompu ou incapable de transformer les expériences inégalitaires. Les études présentées ici retracent les parcours d'engagement de croyants s'inscrivant dans différents courants théologiques, de la mouvance frériste proche de Tariq Ramadan, à des salafis

${ }^{1}$ Cf. Hajjat, (A.), La marche pour l'égalité et contre le racisme, Paris, Editions Amsterdam, 1983. 
revendiqués et des acteurs refusant d'inscrire dans des courants déterminés des préoccupations qui ne sont pas d'abord religieuses.

Ces travaux indiquent l'extrême diversité des mobilisations de musulmans, qui ne fait que refléter l'hétérogénéité sociale de ces acteurs et les multiples formes d'interactions dans lesquelles ils sont insérés. Les formes d'engagement décrites ici oscillent entre des mobilisations contre l'exclusion de l'accompagnement des sorties scolaires, pour le port du voile à l'école, l'organisation d'activités d'éducation populaire et des investissements du jeu politique local ou national. Nous avons à ce titre souhaité inclure un chapitre portant sur l'investissement public des musulmans en Belgique, cas qui indique qu'en dépit d'une apparente proximité de contexte une gestion très différenciée de l'investissement public des musulmans peut se faire jour. Ce chapitre révèle, en creux, les difficultés de l'organisation collective des musulmans en France. Quand de telles initiatives se font jour elles sont bien souvent délégitimées comme impropres à la matrice républicaine hexagonale, de telles critiques étant bien souvent anticipées par les acteurs afin de se prémunir et éviter l'échec de mobilisations hautement improbables. Ces difficultés renforcent l'exclusion sociale et politique que subissent une partie des communautés musulmanes, sapant les bases possibles d'une politisation par l'action collective suscitée par un sentiment d'injustice partagé. Quand bien même le référent religieux ne peut être l'unique support d'engagement des classes populaires racisées, les obstacles rencontrés contribuent à la marginalisation de segments entiers de la population. Rien ne dit en outre, comme l'indiquent certains des chapitres qui suivent, qu'entrer en politique par le religieux confine à cette sphère et ne constitue pas à l'inverse un tremplin vers d'autres combats. Laisser une place à de telles mobilisations et formes de représentation, loin de contribuer à la " communautarisation » de la société, peut à l'inverse constituer un puissant vecteur d'égalité. Ce sont les conditions sociales de tels parcours qui sont abordées dans les pages qui suivent, tout comme leurs devenirs fragiles et leurs conséquences politiques encore indéterminées. 